\title{
Stunning Popularity of LARCs With Good Access and Quality: A Major Opportunity to Meet Family Planning Needs
}

Given true choice, a very high proportion of women, perhaps most, would select one of the long-acting reversible contraceptives (LARCs) - implants or IUDs - for contraception. If implemented on a wide scale, it would not only drastically alter the current method mix but also serve client needs much better and prevent unintended pregnancy more successfully.

See related articles by Curry, Part I; Curry, Part II; and Ross.

There is no such thing as a precisely optimal contraceptive method mix. Individual needs and preferences vary widely, as do cultures and stage of reproductive life. But what might the method mix look like if women and couples were really provided with good choice from among a full range of methods? The Contraceptive CHOICE project in the United States found that a majority of 2,500 young women chose to use one of the long-acting reversible contraceptive (LARC) methods of either implants or intrauterine devices (IUDs). ${ }^{1}$

Now also witness the striking findings from a very different setting-CARE's very large family planning intervention in 5 crisis-affected countries, reported in this issue of GHSP. ${ }^{2,3}$ In this well-executed substantial program, in which more than 52,000 women who were given a wide choice of methods started using a modern method, overall a remarkably high $61 \%$ of them selected LARCs (Figure). To appreciate fully this high proportion, we can compare it to the extensive global data on method mix compiled by Ross et al., also in this issue of GHSP. ${ }^{4}$ Overwhelmingly in low- and middle-income countries, especially in sub-Saharan Africa, shortacting methods by far predominate.

Why this difference? It seems inescapable that short-acting methods predominate because they are by far the most available in most settings. Providing pills, condoms, and injectables is relatively easy, but it is not so easy to provide LARCs and permanent methods. Still, IUDs are widely used in countries such as Egypt and Viet Nam, and sterilization is highly used in India and Latin America. And we have seen that, with robust service delivery models such as mobile outreach and social franchising, use of implants is highly acceptable in Africa. ${ }^{5}$ So programs can successfully provide such methods.
Granted, it may well be true that women in crisisaffected situations are more interested in preventing pregnancy for a longer time. However, provision of LARCs in the CARE program was actually constrained somewhat. In Pakistan, implants provision was impaired by difficulty in securing supply and gaining government approval for community health workers

FIGURE. Method Mix Among New Modern Method Users at CARE-Supported Facilities in 5 Crisis-Affected Countries, a July 2011December 2013

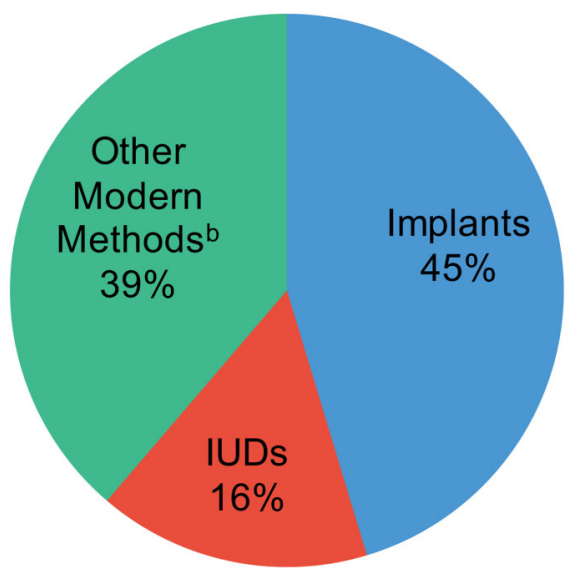

a Chad, Democratic Republic of the Congo, Diibouti, Mali, and Pakistan.

b Other modern methods included injectables, oral contraceptive pills, tubal ligation, and vasectomy. 
(Lady Health Visitors) to insert implants. In Djibouti, training to provide implants and IUDs was delayed. And availability of sterilization was limited overall. Moreover, bear in mind the CARE data are on method selection for new clients. Over time, the proportion and prevalence of LARC use among that same set of women will increase, because continuation of LARCs is much better than that of short-acting methods. Notably, the proportion of new clients selecting IUDs increased progressively over time in crisis settings. And good availability of permanent methods would likely shift the method mix even more.

This all presents a major opportunity. Providing a more balanced method mix, by improving access to long-acting methods, allows family planning programs to meet clients' individual needs much better. And long-acting methods generally enable more successful contraception than short-acting methods, with much lower failure rates and far better continuation rates. Thus, a more optimal method mix will prevent unintended pregnancy better and increase the health benefits of healthy timing and spacing of pregnancy.

The program mandate is clear-priority for well-executed service delivery models, such as mobile outreach, social franchising, and other When provided approaches, for robust, quality provision of with a wide choice LARCs and permanent methods, in the context of methods, of choice among a broad range of methods. -Global Health: Science and Practice

$61 \%$ of women in crisis settings selected LARCs.

\section{REFERENCES}

1. Secura GM, Allsworth JE, Madden T, Mullersman JL, Peipert JF. The Contraceptive CHOICE Project: reducing barriers to longacting reversible contraception. Am J Obstet Gynecol. 2010;203(2):115.e1-7. CrossRef. Medline

2. Curry DW, Rattan J, Nzau JJ, Giri K. Delivering high-quality family planning services in crisis-affected settings I: program implementation. Glob Health Sci Pract. 2015;3(1):14-24 CrossRef

3. Curry DW, Rattan J, Huang S, Noznesky E. Delivering highquality family planning services in crisis-affected settings II: results. Glob Health Sci Pract. 2015;3(1):25-33. CrossRef

4. Ross J, Keesbury J, Hardee K. Trends in the contraceptive method mix in low- and middle-income countries: analysis using a new "average deviation" measure. Glob Health Sci Pract. 2015;3(1):34-55. CrossRef

5. Duvall S, Thurston $S$, Weinberger $M$, Nuccio O, FuchsMontgomery N. Scaling up delivery of contraceptive implants in sub-Saharan Africa: operational experiences of Marie Stopes International. Glob Health Sci Pract. 2014:2(1):72-92. CrossRef. Medline

Cite this article as: Stunning popularity of LARCs with good access and quality: a major opportunity to meet family planning needs. Glob Health Sci Pract. 2015;3(1):12-13. http://dx.doi.org/10.9745/GHSP-D-15-00044.

(c) Global Health: Science and Practice. This is an open-access article distributed under the terms of the Creative Commons Attribution License which permits unrestricted use, distribution, and reproduction in any medium, provided the original author and source are properly cited. To view a copy of the license, visit http://creativecommons.org/licenses/by/3.0/. When linking to this article, please use the following permanent link: http://dx.doi.org/10.9745/GHSP-D-15-00044. 